\title{
Antimicrobial resistance predicts death in Tanzanian children with bloodstream infections: a prospective cohort study
} Bjørn Blomberg*1,2,3, Karim P Manji ${ }^{4}$, Willy K Urassa ${ }^{5}$, Bushir S Tamim ${ }^{4}$, Davis SM Mwakagile ${ }^{5}$, Roland Jureen ${ }^{1,6}$, Viola Msangi ${ }^{5}$, Marit G Tellevik ${ }^{1,2}$, Mona Holberg-Petersen ${ }^{7,8}$, Stig Harthug ${ }^{1,2}$, Samwel Y Maselle ${ }^{5}$ and Nina Langeland ${ }^{1,2}$

\author{
Address: ${ }^{1}$ Department of Medicine, Haukeland University Hospital, Bergen, Norway, ${ }^{2}$ Institute of Medicine, University of Bergen, Norway, ${ }^{3}$ Centre \\ for International Health, University of Bergen, Norway, ${ }^{4}$ Department of Paediatrics and Child Health, Muhimbili University College of Health \\ Sciences, Dar es Salaam, Tanzania, ${ }^{5}$ Department of Microbiology and Immunology, Muhimbili University College of Health Sciences, Dar es \\ Salaam, Tanzania, ${ }^{6}$ Department of Laboratory Medicine, Alexandra Hospital, Singapore, ${ }^{7}$ Department of Microbiology, Ullevål University \\ Hospital, Oslo, Norway and ${ }^{8}$ Faculty of Medicine, University of Oslo, Norway \\ Email: Bjørn Blomberg* - bjorn.blomberg@med.uib.no; Karim P Manji - kmanji@muchs.ac.tz; Willy K Urassa - wurassa@muchs.ac.tz; \\ Bushir S Tamim - tambs2000@yahoo.com; Davis SM Mwakagile - dmwakagile@muchs.ac.tz; Roland Jureen - Roland_Jureen@Alexhosp.com.sg; \\ Viola Msangi - vmsangi@muchs.ac.tz; Marit G Tellevik - marit.gjerde@med.uib.no; Mona Holberg-Petersen - mona.holberg- \\ petersen@ulleval.no; Stig Harthug - stig.harthug@helse-bergen.no; Samwel Y Maselle - smaselle@muchs.ac.tz; \\ Nina Langeland - nina.langeland@helse-bergen.no \\ * Corresponding author
}

Published: 22 May 2007

BMC Infectious Diseases 2007, 7:43 doi:10.1 186/147/-2334-7-43

This article is available from: http://www.biomedcentral.com/I47/-2334/7/43

(c) 2007 Blomberg et al; licensee BioMed Central Ltd.

This is an Open Access article distributed under the terms of the Creative Commons Attribution License (http://creativecommons.org/licenses/by/2.0), which permits unrestricted use, distribution, and reproduction in any medium, provided the original work is properly cited.

\begin{abstract}
Background: Bloodstream infection is a common cause of hospitalization, morbidity and death in children. The impact of antimicrobial resistance and HIV infection on outcome is not firmly established.

Methods: We assessed the incidence of bloodstream infection and risk factors for fatal outcome in a prospective cohort study of 1828 consecutive admissions of children aged zero to seven years with signs of systemic infection. Blood was obtained for culture, malaria microscopy, HIV antibody test and, when necessary, HIV PCR. We recorded data on clinical features, underlying diseases, antimicrobial drug use and patients' outcome.

Results: The incidence of laboratory-confirmed bloodstream infection was I3.9\% (255/1828) of admissions, despite two thirds of the study population having received antimicrobial therapy prior to blood culture. The most frequent isolates were klebsiella, salmonellae, Escherichia coli, enterococci and Staphylococcus aureus. Furthermore, 21.6\% had malaria and $16.8 \%$ HIV infection. One third (34.9\%) of the children with laboratory-confirmed bloodstream infection died. The mortality rate from Gram-negative bloodstream infection (43.5\%) was more than double that of malaria (20.2\%) and Gram-positive bloodstream infection (16.7\%). Significant risk factors for death by logistic regression modeling were inappropriate treatment due to antimicrobial resistance, HIV infection, other underlying infectious diseases, malnutrition and bloodstream infection caused by Enterobacteriaceae, other Gram-negatives and candida.
\end{abstract}

Conclusion: Bloodstream infection was less common than malaria, but caused more deaths. The frequent use of antimicrobials prior to blood culture may have hampered the detection of organisms susceptible to commonly used antimicrobials, including pneumococci, and thus the study probably underestimates the incidence of bloodstream infection. The finding that antimicrobial resistance, HIV-infection and malnutrition predict fatal outcome calls for renewed efforts to curb the further emergence of resistance, improve HIV care and nutrition for children. 


\section{Background}

One in every six African children dies before the age of five years [1]. The World Health Organization (WHO) rank the major causes of mortality in African children younger than five years as neonatal causes (26\%, among which the entity "sepsis or pneumonia" contributes a quarter), pneumonia (21\%), malaria (18\%) diarrhea (16\%) and HIV-infection (6\%) [2]. Bloodstream infection is a frequent cause of morbidity and associated with mortality in excess of 25\% [3]. Since bloodstream infection may occur as part of localized infections with defined foci such as pneumonia and diarrhea, its importance is not reflected in the above estimates of death causes. Bloodstream infection and malaria are practically indistinguishable by clinical examination [4], and available WHO guidelines for managing childhood illnesses fail to identify up to half of the cases of bloodstream infections [5]. A recent study from Kenya [3] found that bloodstream infection caused one quarter of all deaths of children in the hospital, outnumbering malaria deaths. Antimicrobial resistance increases worldwide and does not spare developing countries [6]. However, the impact of antimicrobial resistance on the clinical outcome of infections such as bloodstream infection has been difficult to assess due to a number of factors, including confounding by underlying diseases $[7,8]$. We performed a prospective cohort study to gain knowledge on the etiology and antimicrobial resistance patterns of pediatric bloodstream infections and to identify microbiologic and other risk factors for fatal outcome of these infections.

\section{Methods \\ Location and patients}

The study took place from August 2001 to August 2002 at Muhimbili National Hospital, Dar es Salaam, Tanzania. A total of 1787 children (aged 0-7 years) were consecutively enrolled in a prospective cohort study of 1828 admissions. The inclusion criterion was clinical presentation suspect of systemic infection based on the presence of fever $\left(>=38^{\prime} \mathrm{C}\right)$, hypothermia $\left(<36^{\prime} \mathrm{C}\right)$ and other signs and symptoms as detailed in the WHO's IMCI Integrated Management of Childhood Illness guidelines [9] including general danger signs such as convulsions, lethargy, inability to drink or breastfeed, vomiting, and other signs of infection, such as neck stiffness, bulging fontanelles, cough, tachypnea, difficult breathing, chest in-drawings, nasal flaring, grunting, diarrhea, dehydration, ear or eye discharge, oral thrush, jaundice, enlargement of liver or spleen, lymphadenopathy, and signs of infection in the skin and umbilicus (in neonates). The attending clinician decided on inclusion of the patient and subsequently recorded clinical data using a standardized questionnaire and obtained blood for culture, malaria microscopy and HIV testing. Additionally, patients' medical records and departmental registries for admissions, discharges and deaths were reviewed.

Due to the young age of the study subjects ( $0-7$ years), the parents or other accompanying, responsible family members were asked for written consent on behalf of the patient. Information was given in writing and verbally in the national language, Kiswahili. Written informed consent was obtained before taking blood for microbiological investigations, if feasible. However, in some circumstances, in the case of critically ill patients, blood specimens were taken based on verbal consent, since these investigations are strongly recommended as routine investigations in severely ill, febrile children, and since it would be inappropriate to delay management of such patients due to paperwork. The responsible family member was then approached in retrospect for written consent to use the specimen and information in the study. The responsible family member was allowed to opt out from the HIVtesting and only consent to participation in the blood culture part of the study. As far as possible, the treatment was guided by the test results. In the following, the term "suspected systemic infection" refers to all included patients in the study, and the term "laboratory-confirmed bloodstream infection" refers to growth of one or more clinically relevant bacterial or fungal isolates from bloodculture from a patient who also confirms to the inclusion criterion of suspected systemic infection. Viral and parasitic infections were not included in the definition of bloodstream infection.

Community-acquired infection was defined as bloodstream-infection with growth of pathogenic bacteria in a blood-culture obtained within the first 48 hours after admission. For classification purposes, the time to bloodculture was calculated as the time from admission to the time of receipt of blood culture in the laboratory, allowing for overnight delay in transport for cultures obtained in the evening or at night. A neonate who was born in hospital within the last 10 days was considered as having hospital-acquired infection. If a patient was discharged and re-admitted within ten days, the episode was considered a single admission. The Muhimbili University College of Health Research Ethics Committee approved the study protocol.

\section{Microbiologic methods}

One and five $\mathrm{ml}$ blood from neonates and older children, respectively, were inoculated in BACTEC Myco/F lytic blood-culturing vials (Becton Dickinson, Franklin Lakes, NJ), which supports the growth of fungi and bacteria, including mycobacteria [10]. The blood-cultures were incubated for six weeks. Positive blood-cultures were subcultured on Columbia II agar base (Oxoid Ltd, Basingstoke, UK) with five percent human blood, chocolate agar 
and MacConckey agar (Difco/BD Diagnostic Systems, Sparks, MI, USA). The isolates were identified by standard methods [11], including the use of API20E, API20NE and API 20 AUX systems (bioMérieux SA, Marcy l'Etoile, France). Due to economic limitations, we did not perform anaerobic culture, and, in general, only a single blood culture was taken from each patient. Thus, the study was not designed to evaluate the clinical significance of coagulasenegative staphylococci (CoNS) and bacteria of doubtful or limited pathogenicity. Consequently, CoNS and probable contaminant such as diphteroids, Bacillus species and micrococci were not considered pathogens in this study. Enterococcal isolates were included in the study as pathogens. Cultures with polymicrobial growth were considered clinically relevant if known pathogens were among the constituent isolates.

Susceptibilities against antimicrobial agents were tested by the disk diffusion method according to the Clinical and Laboratory Standards Institute guidelines [12]. Testing for minimum inhibitory concentration (MIC) of antimicrobials was not routinely performed on all isolates. However, MIC determination by E-test (AB Biodisk, Solna, Sweden) was performed for more detailed characterization of the susceptibilities for antimicrobials in Gram-negative bacteria and enterococci. Gram-negative bacteria were investigated for extended-spectrum betalactamases (ESBL) with E-test, PCR and DNA sequencing as described previously [13]. Enterococcal isolates were investigated by PCR to affirm identity (E. faecalis, E. faecium) and to detect vancomycin resistance (van $\mathrm{A}, \operatorname{van} \mathrm{B}$ ). We verified the identity of isolates of $S$. aureus and detected resistance to methicillin/oxacillin with a multiplex PCR targeting the nuc gene and the mecA gene [14].

Malaria testing was performed by microscopy of Giemsastained thick and thin drop blood smears. HIV testing was performed anonymously using a rapid test for HIV1/HIV2 antibodies (ACON HIV 1/2, ACON laboratories, Inc. San Diego, California, USA). HIV antibody-positive sera from children younger than 18 months were analyzed for HIV1 RNA by reverse transcriptase polymerase chain reaction (RT-PCR) using primers targeting the pol gene, JA17 through JA20 [15], and the vif region OG 462/502 [16].

\section{Data analysis}

Data were entered in a database based on the free-ofcharge WHONET software for surveillance of antimicrobial resistance available as a download from the World Health Organization $[17,18]$, and further data management was done in Filemaker Database software. Statistical analysis was performed in Stata 8 (Stata Corporation, College Station, Texas, US). Univariate assessment of risk factors for intra-hospital death was done by Fisher's exact test with a two-sided $P$-value and odds ratios and 95\% confi- dence intervals were obtained by the 'logistic' function in Stata. Significant factors to the level of $P<0.2$ from the univariate analysis and a priori important factors such as sex, age and underlying diseases were included in the multivariate analysis. Multivariate analysis was performed by automated and manual backwards step-wise logistic regression where factors with $P>0.2$ were removed from the model. We present four logistic regression models using different subsets of the study population analyzing cases of laboratory-confirmed bloodstream infection $(\mathrm{n}=$ 216) as well as cases of clinically suspected systemic infection ( $\mathrm{n}=1527)$, and for each category we re-analyzed the data for those who had known HIV status ( $\mathrm{n}=128$ and $\mathrm{n}$ $=790$, respectively). Comparisons of medians of time variables were done by Wilcoxon rank-sum (Mann-Whitney) test.

\section{Results \\ Patients}

A total of 1787 patients with a median age of 8.5 months were admitted 1828 times. Forty-four percent (795/1787) were female. Neonates (median age 3 days, range 0-30) accounted for almost a third of the admissions $(29.3 \%$, $535 / 1828$ ), and stayed a median of 6 days (range 1-53) in the hospital. Older children (median age 1 year, range 1 month -7 years) stayed a median of 7 days (range 1-78) in the hospital.

The clinical outcome was known for $89.3 \%$ (1632/1828) of the admissions. Among the 17.0\% (277/1632) who died in hospital, 22.4\% ( $\mathrm{n}=62)$ had laboratory-confirmed bloodstream infection, $17.7 \%(\mathrm{n}=49)$ had malaria, $6.5 \%(n=18)$ had concomitant laboratory-confirmed bloodstream infection and malaria-parasitemia and $52.0 \%(\mathrm{n}=144)$ had neither. Readmissions comprised $3.2 \%(n=59)$ of all admissions (39 and 1 patient were readmitted once and twice, respectively, 18 patients had been admitted prior to the study).

\section{Antimicrobial therapy}

Information on antimicrobial use was available for $85.2 \%$ (1557/1828) of the admissions. The majority of patients (93.8\%) received antimicrobial therapy (Table 1) and at least two-thirds $(67.2 \%, 1046 / 1557)$ of the patients did so before blood-culture was taken. There was no formal empirical regimen for the treatment of sepsis at the hospital, partly because of the scarcity of local studies on antimicrobial resistance of relevant bacterial isolates. However, most neonates received a regimen of ampicillin + cloxacillin + gentamicin. One-fifth of the neonates received ceftriaxone, in most cases as a second-line regimen in case of unsatisfactory response to the first regimen. In older children there was a more diverse pattern of regimens used, in most cases including one or more penicillins (ampicillin, penicillin G or cloxacillin) and either 
chloramphenicol or gentamicin. To some extent, accompanying localizing signs did impact on the choice of antimicrobial regimen, most notably in the case of suspected meningitis in children older than 1 month when a regimen based on chloramphenicol and a penicillin would often be used, and in the case of bloody diarrhea when erythromycin was often added. Severe malaria was treated with quinine, while less severe cases were treated with chloroquine in neonates and pyrimethamine-sulfadoxin in older children. Oral thrush was treated with nystatin mixture, but systemic antifungal drugs were rarely used. Ciprofloxacin was not used in children at the hospital.

\section{Types of bloodstream infections}

The incidence of laboratory-confirmed bloodstream infection was $13.9 \%$ (255/1828) of all admissions, $15.9 \%$ (85/ 535) among neonates and 13.1\% (170/1293) among older children. A single pathogen was recovered from 224 children $(12.3 \%)$, while $31(1.7 \%)$ had polymicrobial infection with two $(\mathrm{n}=26)$, three $(\mathrm{n}=2)$ or four isolates $(\mathrm{n}=3)$. In total, 294 pathogenic bacterial and fungal isolates were recovered (Table 2). Among all laboratory-confirmed bloodstream infections, half (128/255) were defined as potentially hospital-acquired. Salmonella and
Escherichia coli were the most common isolates in community-acquired infections, and klebsiella and Staphylococcus aureus were the most common in hospital-acquired infections (Table 2). Klebsiella was, by far, the most common cause of neonatal bloodstream infection, particularly in early-onset infection (0-6 days of age) where it responsible for approximately one-third of the cases (Table 3 ). In children older than 1 month of age, salmonellae were the most frequently isolated pathogens (Table 3 ).

Among all children with suspected systemic infection, those with laboratory-confirmed bloodstream infection had three times increased risk of dying (Table 4, Figure 1), and among those who survived, the duration of hospital stay was significantly longer than for children without laboratory-verified bloodstream infection (median 8 versus 6 days, $P<0.001)$. Univariate and multivariate analysis of risk factors for death are shown in Table 4 and 5, respectively. The mortality rate from Gram-negative bloodstream infection $(45.6 \%)$ was more than twice that of malaria $(20.2 \%)$ and Gram-positive bloodstream infection $(16.7 \%)$. Positive blood-culture with Enterobacteriaceae, other Gram-negative bacteria and candida were independent risk factors for fatal outcome (Table 5, Figure

Table I: Use of antimicrobial agents in children with suspected systemic infection

\begin{tabular}{|c|c|c|c|c|}
\hline \multirow[b]{2}{*}{ Age of child on blood culture } & \multicolumn{4}{|c|}{ Number of children (\%) receiving drug } \\
\hline & $0-6$ days & 7-30 days & I month - 7 years & Total \\
\hline No of patients & $n=26 I$ & $n=190$ & $n=1106$ & $n=1557$ \\
\hline Any antibacterial & 257 (98.5) & $186(97.9)$ & $1017(92.0)$ & 1460 (93.8) \\
\hline - Penicillin & - & - & $301(27.2)$ & $301(19.3)$ \\
\hline - Ampicillin/amoxicillin & $244(93.5)$ & $165(86.8)$ & $589(53.3)$ & $998(64.1)$ \\
\hline - Cloxacillin & $244(93.5)$ & $165(86.8)$ & $354(32.0)$ & $763(49.0)$ \\
\hline - Cephalexin & - & - & $27(2.4)$ & $27(1.7)$ \\
\hline - Cefuroxime & - & - & $37(3.3)$ & $37(2.4)$ \\
\hline - Ceftriaxone & $34(13.0)$ & $51(26.8)$ & $118(10.7)$ & $203(13.0)$ \\
\hline - Chloramphenicol & - & $2(1.1)$ & $416(37.6)$ & $418(26.8)$ \\
\hline - Gentamicin & $250(95.8)$ & $173(91.1)$ & $504(45.6)$ & $927(59.5)$ \\
\hline - Amikacin & - & I (0.5) & $25(2.3)$ & $26(1.7)$ \\
\hline - Co-trimoxazole & - & $3(1.6)$ & $129(11.7)$ & $132(8.5)$ \\
\hline - Erythromycin & - & $2(1.1)$ & $44(4.0)$ & $46(3.0)$ \\
\hline - Azithromycin & - & - & $23(2.1)$ & $23(1.5)$ \\
\hline - Nalidixic acid & - & - & $4(0.4)$ & $4(0.3)$ \\
\hline - Metronidazole & - & - & $22(2.0)$ & $22(1.4)$ \\
\hline TB medicines & - & - & $71(6.4)$ & $71(4.6)$ \\
\hline Any antimalarial & $19(7.3)$ & $23(12 . I)$ & 784 (70.9) & $826(53.1)$ \\
\hline - Quinine & $6(2.3)$ & $16(8.4)$ & $636(57.5)$ & $658(42.3)$ \\
\hline - Sulfadoxine-pyrimethamine & - & I (0.5) & $192(17.4)$ & $193(12.4)$ \\
\hline - Chloroquine & $15(5.7)$ & $9(4.7)$ & $7(0.6)$ & $31(2.0)$ \\
\hline - Amodiaquine & - & - & $12(1.1)$ & $12(0.8)$ \\
\hline - Artesunate & - & - & $\mathrm{I}(0.1)^{\prime}$ & $\mathrm{I}(0.1)$ \\
\hline Mebendazole & - & - & $44(4.0)$ & $44(2.8)$ \\
\hline Nystatin (oral) & $8(3.1)$ & $13(6.8)$ & $195(17.6)$ & $216(13.9)$ \\
\hline Systemic antifungal & - & - & $8(0.7)$ & $8(0.5)$ \\
\hline
\end{tabular}


Table 2: Frequency (percentage) of bacterial and fungal pathogens* cultured from community-acquired $\dagger$ and hospital-acquired $\ddagger$ bloodstream infection

\begin{tabular}{|c|c|c|c|}
\hline Organism & CAt & HA $\ddagger$ & Total \\
\hline Klebsiella spp. & $19(12.3)$ & $34(24.5)$ & $53(18.0)$ \\
\hline - Klebsiella pneumoniae & 17 & 31 & 48 \\
\hline Salmonellae & $27(17.4)$ & $12(8.6)$ & $39(13.3)$ \\
\hline - Salmonella serovar Typhii & 1 & i & 2 \\
\hline - Salmonella serovar Enteritidis & 14 & 6 & 20 \\
\hline - Salmonella serovar Typhimurium & 11 & 5 & 16 \\
\hline - Salmonella serovar Newport & I & 0 & I \\
\hline Escherichia coli & $24(15.5)$ & $13(9.4)$ & $37(12.6)$ \\
\hline Enterobacter spp. & $5(3.2)$ & $4(2.9)$ & $9(3.1)$ \\
\hline - Enterobacter cloacae & 2 & 4 & 6 \\
\hline - Other Enterobacter spp. & 3 & 0 & 3 \\
\hline Other Enterobacteriaceae & $4(2.6)$ & $2(1.4)$ & $6(2.0)$ \\
\hline - Pantoea spp. & 2 & 0 & 2 \\
\hline - Serratia marcescens & 0 & I & I \\
\hline - Shigella flexnerii & 1 & 0 & I \\
\hline - Citrobacter freundii & 1 & 0 & 1 \\
\hline - Proteus mirabilis & 0 & I & I \\
\hline Total Enterobacteriaceae & $79(51.0)$ & $65(46.8)$ & $144(49.0)$ \\
\hline Acinetobacter spp. & $3(1.9)$ & $9(6.5)$ & $12(4.1)$ \\
\hline - Acinetobacter baumannii & 0 & 5 & 5 \\
\hline - Acinetobacter Iwoffii & 3 & 2 & 5 \\
\hline - Acinetobacter spp. & 0 & 2 & 2 \\
\hline Pseudomonas aeruginosa & $7(4.5)$ & $6(4.3)$ & $13(4.4)$ \\
\hline Other Non-Enterobacteriaceae & $6(3.9)$ & $5(3.6)$ & II (3.7) \\
\hline Pseudomonas spp. & 4 & 1 & 5 \\
\hline Sphingomonas paucimobilis & 2 & 0 & 2 \\
\hline Chryseobacterium spp. & 0 & 2 & 2 \\
\hline Moraxella spp. & 0 & 2 & 2 \\
\hline Total non-EnterobacteriaceaeGNR & $16(10.3)$ & $20(14.4)$ & $36(12.2)$ \\
\hline GNR not further specified & $5(3.2)$ & $2(1.4)$ & $7(2.4)$ \\
\hline Total GNR & $100(64.5)$ & $87(62.6)$ & $187(63.6)$ \\
\hline Staphylococcus aureus & $13(8.4)$ & $17(12.2)$ & $30(10.2)$ \\
\hline Enterococci & $24(15.5)$ & $19(13.7)$ & $43(14.6)$ \\
\hline - Enterococcus faecium & 12 & 9 & 21 \\
\hline - Enterococcus faecalis & 9 & 6 & 15 \\
\hline - Enterococcus spp. & 3 & 4 & 7 \\
\hline Streptococci & $3(1.9)$ & $5(3.6)$ & $8(2.7)$ \\
\hline - Group B streptococci & 1 & 3 & 4 \\
\hline - Streptococcus viridans & I & 2 & 3 \\
\hline - Streptococci, not further identified & I & 0 & 1 \\
\hline Total Gram-positive & $40(25.8)$ & $41(29.5)$ & 81 (27.6) \\
\hline Mycobacterium tuberculosis & $0(0.0)$ & I (0.5) & I $(0.3)$ \\
\hline Candida spp. & $15(9.7)$ & $10(7.2)$ & $25(8.5)$ \\
\hline Total & $155(100.0)$ & $139(100.0)$ & $294(100.0)$ \\
\hline
\end{tabular}

GNR, Gram-negative rods. *The percentage refers to the proportion of all pathogenic bacterial and fungal isolates. Anaerobes, coagulase-negative staphylococci and bacterial isolates of uncertain pathogenicity were not included. $\dagger$ CA: Community-acquired infection, i.e. blood-culture obtained $\leq 48$ hours from of admission. $\ddagger$ Hospital-acquired infection: Blood-culture obtained $>48$ hours from admission.

2). In a separate logistic regression model examining only the pathogen involved, the significant risk factors for death were, in descending order of odds ratio, growth of Enterobacter spp. (odds ratio 10.5, $P=0.007$ ), Pseudomonas aeruginosa (9.7, $P<0.001)$, Salmonella Typhimurium (6.4, $P=0.001)$, E. coli $(5.0, P<0.001)$, klebsiella $(4.5, P<$ 0.001 ), and candida $(2.5, P=0.049)$ (data not shown in table). Growth of Salmonella Enteritidis, Acinetobacter spp.,
E. faecalis, E. faecium and S. aureus were not significant risk factors for death.

\section{Malaria}

Malaria slides were positive in $21.6 \%$ (354/1638). Malaria parasitemia was neither associated with HIVinfection $(P=0.227)$, nor bloodstream infection caused by any pathogen $(P=0.730)$, Gram-negative bacteria $(P=$ 
Table 3: Episodes of laboratory-confirmed bloodstream infection in different age groups of children

\begin{tabular}{|c|c|c|c|c|}
\hline Organism* & Early onset $\uparrow \mathbf{N}(\%)$ & Late onset $\ddagger \mathbf{N}(\%)$ & Older§ N (\%) & Total N (\%) \\
\hline Klebsiella spp. & $17(3 \mid .5)$ & $7(22.6)$ & $12(7.1)$ & $36(14.1)$ \\
\hline Salmonellae & $0(0.0)$ & I (3.2) & $32(18.8)$ & $33(12.9)$ \\
\hline Escherichia coli & $6(11.1)$ & $3(9.7)$ & $19(11.2)$ & $28(11.0)$ \\
\hline Enterobacter spp. & $4(7.4)$ & $\mathrm{I}(3.2)$ & $2(1.2)$ & $7(2.7)$ \\
\hline Other Enterobacteriaceae & I (I.9) & $0(0.0)$ & I (0.6) & $2(0.8)$ \\
\hline Total Enterobacteriaceae & $28(51.9)$ & 12 (38.7) & $66(38.8)$ & $106(41.6)$ \\
\hline Acinetobacter spp. & I (I.9) & $0(0.0)$ & $10(5.9)$ & $11(4.3)$ \\
\hline Pseudomonas aeruginosa & $0(0.0)$ & $0(0.0)$ & $9(5.3)$ & $9(3.5)$ \\
\hline Other non-Enterobacteriaceae & $4(7.4)$ & $0(0.0)$ & $5(2.9)$ & $9(3.5)$ \\
\hline Total non-Enterobacteriaceae & $5(9.3)$ & $0(0.0)$ & $24(14.1)$ & $29(11.4)$ \\
\hline GNR not further specified & I (1.9) & I (3.2) & $5(2.9)$ & $7(2.7)$ \\
\hline Total GNR & $34(63.0)$ & $13(41.9)$ & $95(55.9)$ & $142(55.7)$ \\
\hline Staphylococcus aureus & $6(11.1)$ & $5(16.1)$ & $15(8.8)$ & $26(10.2)$ \\
\hline Enterococci & $3(5.6)$ & $4(12.9)$ & $23(13.5)$ & $30(11.8)$ \\
\hline Group B streptococci & $2(3.7)$ & I (3.2) & $0(0.0)$ & $3(1.2)$ \\
\hline Other Streptococcus & I (I.9) & $0(0.0)$ & $2(1.2)$ & $3(1.2)$ \\
\hline Total Gram positive & $12(22.2)$ & $10(32.3)$ & $40(23.5)$ & $62(24.3)$ \\
\hline Mycobacterium tuberculosis & $0(0.0)$ & $0(0.0)$ & I (0.6) & I (0.4) \\
\hline Candida spp. & I (I.9) & $3(9.7)$ & $15(8.8)$ & $19(7.5)$ \\
\hline Polymicrobial infections & $7(13.0)$ & $5(16.1)$ & 19 (11.2) & $31(12.2)$ \\
\hline Total & $54(100.0)$ & $31(100.0)$ & $170(100.0)$ & $255(100.0)$ \\
\hline
\end{tabular}

GNR, Gram-negative rods. *The percentage refers to the proportion of all episodes of laboratory-confirmed bloodstream infection. Anaerobes, coagulase-negative staphylococci and bacterial isolates of uncertain pathogenicity were not included. $\dagger$ Early-onset $=$ age $0-6$ days. $\neq$ Late-onset $=$ age $7-30$ days. + Older $=1$ month -7 years.

0.296), Enterobacteriaceae $(P=0.351)$, E. coli $(P=0.516)$, klebsiella $(P=1.000)$, non-typhoid salmonella $(P=$ $0.664)$. The case-fatality rate was not significantly higher in patients with malaria parasitemia $(20.2 \%)$ compared to those without $(16.4 \%, P=0.102)$. However, in patients with positive malaria slides, mortality was almost three times higher among those with concomitant Gram-negative bloodstream infection $(45.7 \%$ versus $17.2 \%, P<$ 0.001).

\section{Susceptibility to antimicrobial agents}

Enterobacteriaceae displayed high rates of resistance to commonly used antimicrobials (Table 6). Only $20 \%$ of community-acquired Enterobacteriaceae isolates was sensitive to ampicillin, while two-thirds were sensitive to gentamicin. Two-thirds of community-acquired salmonellae were sensitive to chloramphenicol, while half are resistant to ampicillin and to co-trimoxazole. Isolates of Enterobacteriaceae were almost uniformly sensitive to ciprofloxacin. $P$. aeruginosa isolates were commonly sensitive to antipseudomonas drugs such as ceftazidime, ciprofloxacin and tobramicin (Table 7). ESBL phenotype was found in $18 \%$ of the Enterobacteriaceae isolates (E. coli $9 / 37$, klebsiella 9/53, Enterobacter spp. 5/9, salmonella 1/39 and Pantoea spp 2/2) involving TEM-63, SHV-2a, SHV-12 and CTX-M-15 genotypes [13], and in 3 isolates of non-Enterobacteriaceae (one Acinetobacter spp. and the 2 Chryseobacterium spp.). ESBL-producing isolates were resistant to almost all tested antimicrobials except for ciprofloxacin and meropenem.

The majority of $S$. aureus isolates were sensitive to commonly used anti-staphylococcal agents, including cloxacillin and gentamicin (Table 8). Among the three isolates phenotypically resistant to oxacillin, only one was available for confirmatory detection of the mecA gene by PCR. The child with confirmed MRSA genotype died, the two other survived. Ten of 21 E. faecium isolates showed combined resistance to ampicillin and gentamicin (highlevel), but only one patient with bloodstream infection caused by these organisms died. This combined resistance trait occurred in both community-acquired (4/12) and hospital-acquired infections (6/9), and the difference was not statistically different $(P=0.198)$. Six of $15 E$. faecalis isolates were high-level gentamicin-resistant (but not ampicillin-resistant) and were involved in the death of two patients, among whom one had community-acquired infection. The majority of candida isolates were susceptible to fluconazole $(96 \%)$ and amphotericin B (87\%).

Antimicrobial treatment prior to blood culture was significantly associated with resistance to co-trimoxazole $(83 \%$ versus $69 \%, P=0.036)$ and chloramphenicol (59\% versus $42 \%, P=0.035)$ in Gram-negative isolates as a group, with resistance to erythromycin (36\% versus $0 \%, P=$ 0.014 ) and chloramphenicol ( $46 \%$ versus $0 \%, P=0.005$ ) 
Table 4: Univariate analysis of risk factors for intrahospital death among 1632 children* with suspected systemic infection

\begin{tabular}{|c|c|c|c|c|}
\hline Risk factor & CFR \% (n) & OR & $95 \% \mathrm{Cl}$ & $P$ \\
\hline Overall & $17.0(277 / 1632)$ & & & \\
\hline Male sex & $15.5(143 / 920)$ & 0.79 & 0.61 to 1.03 & 0.084 \\
\hline Neonate $(<\mathrm{I} \mathrm{m})$ & $14.8(70 / 473)$ & 0.80 & 0.59 to 1.07 & 0.146 \\
\hline \multicolumn{5}{|l|}{ Underlying conditions } \\
\hline Malnutrition & $29.3(71 / 242)$ & 2.37 & 1.73 to 3.25 & $<0.001$ \\
\hline HIV infection (laboratory confirmed) & $31.7(46 / 145)$ & 2.40 & 1.60 to 3.59 & $<0.001$ \\
\hline Other infectious underlying conditions & $30.6(33 / 108)$ & 2.29 & 1.48 to 3.52 & $<0.001$ \\
\hline - Tuberculosis & $30.5(29 / 95)$ & 2.26 & 1.43 to 3.58 & 0.001 \\
\hline - Hepatitis & $44.4(4 / 9)$ & 3.92 & 1.04 to 14.67 & 0.052 \\
\hline - Congenital syphilis & $50.0(1 / 2)$ & 4.85 & 0.30 to 77.79 & 0.313 \\
\hline Other non-infectious underlying conditions & $17.1(20 / 117)$ & 1.00 & 0.60 to 1.64 & 1.000 \\
\hline - Sickle cell disease & $3.0(1 / 33)$ & 0.15 & 0.02 to 1.09 & 0.032 \\
\hline - Neoplasia & $60.0(3 / 5)$ & 7.32 & 1.22 to 44.05 & 0.038 \\
\hline - Congenital heart disease & $27.3(9 / 33)$ & 1.77 & 0.82 to 3.83 & 0.156 \\
\hline Malaria (laboratory confirmed) & $20.2(67 / 331)$ & 1.29 & 0.95 to 1.76 & 0.102 \\
\hline \multicolumn{5}{|l|}{ Growth in blood culture of: } \\
\hline Any pathogen & $34.9(80 / 229)$ & 3.29 & 2.41 to 4.48 & $<0.001$ \\
\hline Any GNR & $43.5(64 / 147)$ & 4.60 & 3.22 to 6.60 & $<0.001$ \\
\hline Any Enterobacteriaceae & $45.6(52 / 114)$ & 4.82 & 3.25 to 7.15 & $<0.001$ \\
\hline - E. coli & $50.0(15 / 30)$ & 5.11 & 2.47 to 10.59 & $<0.001$ \\
\hline - Klebsiella spp. & $48.9(22 / 45)$ & 5.00 & 2.74 to 9.10 & $<0.001$ \\
\hline - All non-typhoid Salmonellae & $36.4(12 / 33)$ & 2.88 & 1.40 to 5.92 & 0.007 \\
\hline - Salmonella Enteritidis & $21.1(4 / 19)$ & 1.31 & 0.43 to 3.97 & 0.548 \\
\hline - Salmonella Typhimurium & $53.9(7 / 13)$ & 5.83 & 1.94 to 17.48 & 0.003 \\
\hline - Enterobacter spp. & $71.4(5 / 7)$ & 12.43 & 2.40 to 64.43 & 0.002 \\
\hline Any non-Enterobacteriaceae GNR & $40.6(13 / 32)$ & 3.46 & 1.69 to 7.10 & 0.001 \\
\hline - Pseudomonas aeruginosa & $66.7(8 / 12)$ & 10.04 & 3.00 to 33.59 & $<0.001$ \\
\hline - Acinetobacter spp. & $9.1(1 / 11)$ & 0.49 & 0.06 to 3.82 & 0.702 \\
\hline Gram-positive pathogent & $16.7(11 / 66)$ & 0.98 & 0.50 to 1.89 & 1.000 \\
\hline - Enterococci & $19.4(7 / 36)$ & 1.19 & $0.5 \mathrm{I}$ to 2.73 & 0.655 \\
\hline - E. faecium & $6.7(1 / 15)$ & 0.35 & 0.05 to 2.65 & 0.490 \\
\hline - E. faecalis & $28.6(4 / 14)$ & 1.97 & 0.61 to 6.33 & 0.275 \\
\hline - S. aureus & $7.1(2 / 28)$ & 0.37 & 0.09 to 1.58 & 0.208 \\
\hline Candida & $33.3(8 / 24)$ & 2.49 & 1.05 to 5.87 & 0.049 \\
\hline Polymicrobial infection & $41.4(12 / 29)$ & 3.56 & 1.68 to 7.55 & 0.002 \\
\hline \multicolumn{5}{|l|}{ Inappropriate antimicrobial therapy due to: } \\
\hline - ESBL-producing, multiresistant isolates & $71.4(15 / 21)$ & 12.87 & 4.95 to 33.48 & $<0.001$ \\
\hline - Other bacterial resistance (non-ESBL) & $37.8(17 / 45)$ & 3.14 & 1.69 to 5.83 & 0.001 \\
\hline - Any bacterial resistance (ESBL + other) & $47.7(31 / 65)$ & 5.01 & 3.02 to 8.31 & $<0.001$ \\
\hline - Any antimicrobial resistance (including fungi) & $43.8(39 / 89)$ & 4.39 & 2.82 to 6.83 & $<0.001$ \\
\hline Hospital-acquired infection & $35.5(44 / / 24)$ & 3.01 & 2.03 to 4.46 & $<0.001$ \\
\hline Readmission stay & $15.2(14 / 92)$ & 0.87 & 0.49 to 1.56 & 0.775 \\
\hline
\end{tabular}

CFR, case-fatality rate; OR, odds ratio; $95 \% \mathrm{Cl}$ : 95\% confidence interval; $P$, p-value calculated with two-sided Fisher's exact test; GNR, Gramnegative rod; ESBL, extended-spectrum beta-lactamase. *The outcome was known for 1632 among a total of 1828 admissions. $\dagger$ Anaerobes, coagulase-negative staphylococci and bacterial isolates of uncertain pathogenicity were not included. $\ddagger$ Blood culture obtained $>2$ days after admission. 
Table 5: Logistic regression analysis of risk factors for intra-hospital death from laboratory-confirmed bloodstream infection and clinically suspected systemic infection

\begin{tabular}{|c|c|c|c|c|}
\hline \multirow{2}{*}{$\begin{array}{l}\text { Characteristic } \\
\text { HIV-status analyzed }\end{array}$} & \multicolumn{2}{|c|}{ Laboratory-confirmed bloodstream infection } & \multicolumn{2}{|c|}{ Clinically suspected systemic infection } \\
\hline & No & Yes & No & Yes \\
\hline \multirow[t]{2}{*}{ No. of observations } & $n=216$ & $n=128$ & $n=1527$ & $n=790$ \\
\hline & OR $(95 \% \mathrm{Cl})$ & OR $(95 \% \mathrm{Cl})$ & OR $(95 \% \mathrm{Cl})$ & OR $(95 \% \mathrm{Cl})$ \\
\hline Male sex & $*$ & * & $0.8(0.60-1.05)$ & * \\
\hline Neonate $(\leq \mathrm{I}$ month) & $*$ & $*$ & * & $0.7(0.45-1.15)$ \\
\hline Malnutrition & $1.9(0.95-3.88)$ & $*$ & $2.1(1.47-2.90) \ddagger$ & $1.6(0.96-2.55)$ \\
\hline HIV infection & NA & $3.4(1.22-9.40) \dagger$ & NA & $2.1(1.29-3.26) \dagger$ \\
\hline Other underlying infectious disease & $*$ & * & $1.8(1.13-2.87) \dagger$ & * \\
\hline Underlying non-infectious disease & $*$ & $*$ & * & $*$ \\
\hline Malaria & $*$ & $*$ & * & $*$ \\
\hline Hospital-acquired infection & $*$ & * & * & * \\
\hline Polymicrobial infection & $*$ & $*$ & $*$ & $*$ \\
\hline \multicolumn{5}{|l|}{ Growth in blood-culture of: } \\
\hline - Enterobacteriaceae & $3.5(1.7 \mathrm{I}-7.03) \dagger$ & $5.6(2.06-14.95) \dagger$ & $3.3(2.09-5.34) \ddagger$ & $4.2(2.27-7.65) \ddagger$ \\
\hline - Non -Enterobacteriaceae & $2.4(0.92-6.13)$ & $4.0(0.99-16.45)$ & $2.4(1.02-5.46) \dagger$ & $2.7(0.80-8.81)$ \\
\hline - Candida spp. & $2.6(0.91-7.29)$ & $2.9(0.70-12.09)$ & $2.5(1.02-6.03) \dagger$ & $*$ \\
\hline Inappropriate antimicrobial treatment & $2.1(1.09-4.16) \dagger$ & $2.3(0.95-5.58)$ & $2.1(1.14-3.93) \dagger$ & $1.7(0.76-3.73)$ \\
\hline
\end{tabular}

OR, odds ratio; $95 \% \mathrm{Cl}, 95 \%$ confidence interval. *Variables with $P>0.2$ were removed from the models. Statistically significant risk factors are marked $\dagger P<0.05$ and $\ddagger P<0.001$.

in S. aureus and chloramphenicol in E. faecalis ( $89 \%$ versus $20 \%, P=0.023$ ). In addition, all three MRSA isolates were from patients who had received previous antimicrobial treatment, but this was not statistically significant.

Hospital-acquisition of infection was significantly associated with resistance to amoxicillin-clavulanate and cephalosporins in E. coli and with co-trimoxazole-resistance in

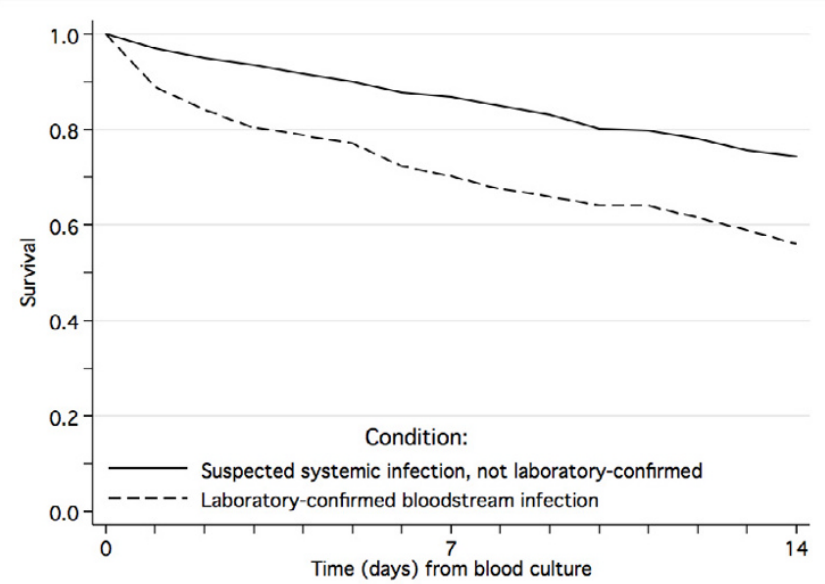

\section{Figure I}

Survival from laboratory-confirmed bloodstream infection and clinically suspected systemic infection. klebsiella. The percentage of bloodstream infection episodes caused by an ESBL-producing organism was not significantly different among community-acquired (7\%, 9/ $127)$ and hospital-acquired infections $13 \%(16 / 128, P=$ 0.206 ). Patients with hospital-acquired laboratory-verified bloodstream infection did not receive inappropriate antimicrobial treatment more often $(41 \%)$ than those with community-acquired infection $(42 \%, P \cong 0.892)$.

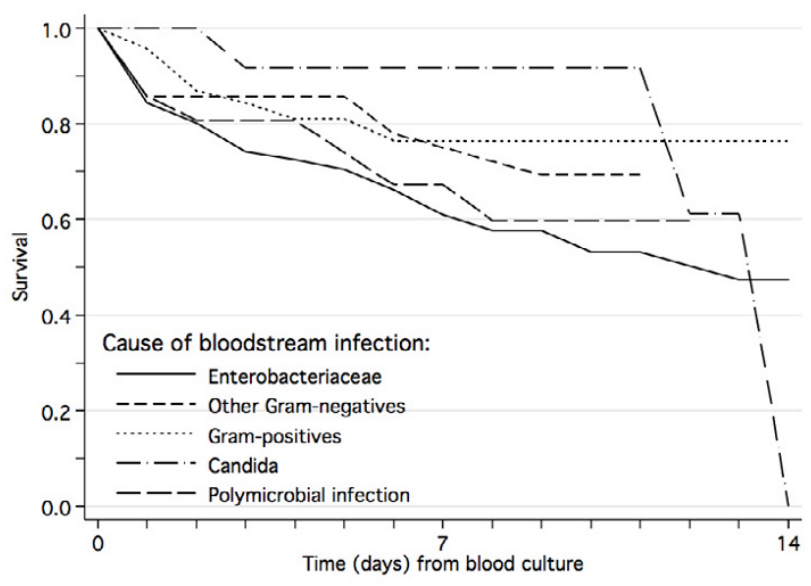

Figure 2

Impact of causative organism on survival from laboratoryconfirmed bloodstream infection. 
Table 6: Antimicrobial susceptibility (percentage) of Enterobacteriaceae isolates causing bloodstream infection

\begin{tabular}{|c|c|c|c|c|c|c|c|c|c|c|}
\hline \multirow[t]{2}{*}{ Antimicrobial } & \multicolumn{2}{|c|}{ Klebsiella } & \multicolumn{2}{|c|}{ Salmonella } & \multicolumn{2}{|c|}{ E. coli } & \multicolumn{2}{|c|}{ Other* } & \multicolumn{2}{|c|}{ Total } \\
\hline & CA 19 & HA 34 & CA 27 & $\mathrm{HA} 12$ & CA 24 & HA 13 & CA 9 & HA 6 & CA 79 & HA 65 \\
\hline Ampicillin & 0 & 0 & 52 & 33 & 4 & 15 & 11 & 33 & 20 & 12 \\
\hline Amoxicillin-clavulanate & 53 & 62 & 70 & 67 & 75 & $31 \dagger$ & 11 & 17 & 61 & 52 \\
\hline Cefuroxime & 74 & 85 & 93 & 100 & 88 & $54 \dagger$ & 56 & 50 & 82 & 78 \\
\hline Ceftazidime & 79 & 85 & 96 & 100 & 88 & $46 \dagger$ & 56 & 50 & 85 & 77 \\
\hline Cefotaxime & 78 & 85 & 96 & 100 & 88 & $50 \dagger$ & 56 & 67 & 84 & 80 \\
\hline Meropenem & 100 & 100 & 100 & 100 & 100 & 100 & 100 & 100 & 100 & 100 \\
\hline Gentamicin & 53 & 53 & 74 & 67 & 71 & 54 & 33 & 50 & 63 & 55 \\
\hline Doxycycline & 58 & 71 & 85 & 75 & 13 & 23 & 33 & 17 & 51 & 57 \\
\hline Co-trimoxazole & 37 & $6 \dagger$ & 52 & 33 & 13 & 23 & 22 & 50 & 33 & 18 \\
\hline Chloramphenicol & 47 & 56 & 85 & 67 & 33 & 46 & 22 & 50 & 53 & 55 \\
\hline Ciprofloxacin & 100 & 100 & 100 & 100 & 92 & 92 & 89 & 100 & 96 & 98 \\
\hline
\end{tabular}

CA, community-acquired infection; HA, hospital-acquired infection. *Other isolates include Enterobacter spp., (9), Pantoea spp. (2), Serratia marcescens (I), Shigella flexneri (I), Citrobacter freundii (I), Proteus mirabilis (I). † Statistically significant difference in susceptibility among hospitalacquired and community-acquired isolates, $\mathrm{p}<0.05$ by two-sided Fisher's exact test.

Inappropriate antimicrobial treatment was a risk factors for death irrespectively of whether controls were patients with clinically suspected infection or laboratory-confirmed bloodstream infection (Table 5, Figure 3). In those who survived, inappropriate antimicrobial treatment for the bloodstream-infection was associated with increased duration of hospital stay (median 8 days versus 6 days, $P$ $=0.043$ ).

Hospital-acquired infection was associated with increased duration of hospitalization (median 10 days versus 6 days, $P<0.001$ ), but was not a significant risk factor for fatal outcome in the multivariate analysis (Table 5, Figure $4)$.

\section{HIV-infection}

Fifty-one percent $(911 / 1787)$ of the study subjects were examined for HIV antibodies and 24.0\% (219/911) were positive. The HIV-1 PCR was positive in $55.4 \%(82 / 148)$ of children younger than 18 months and $8.9 \%(5 / 56)$ of neonates. Thus, the HIV-1 prevalence was $1.7 \%$ (5/302) for neonates, $24.4 \%$ (147/603) for older children, and the combined overall prevalence was $16.8 \%$ (152/905).

Laboratory-confirmed bloodstream infection was not significantly more frequent in HIV-positive children (17.5, $28 / 160)$ than in HIV-negative ones $(15.5 \%, 119 / 766, P=$ 0.552 ). However, bloodstream infection was more frequently caused by non-typhoid salmonella in HIV-positive children $(25 \%, 7 / 28)$ than in HIV-negative children $(8.4 \%, 10 / 119, P=0.022)$. There was no such association between HIV-infection and bloodstream infection caused by E. coli ( $17.9 \%$ versus $10.9 \%, P=0.339)$, ESBL-producing organisms $(17.9 \%$ versus $10.1 \%, P=0.321)$, candida ( $10.7 \%$ versus $10.9 \%, P=1.000)$ or other pathogens, except for bloodstream infection caused by klebsiella,

Table 7: Antimicrobial susceptibility (percentage) of non-Enterobacteriaceae Gram-negative isolates causing bloodstream infection

\begin{tabular}{|c|c|c|c|c|c|c|c|c|}
\hline \multirow[t]{2}{*}{ Antimicrobial } & \multicolumn{2}{|c|}{ P. aeruginosa } & \multicolumn{2}{|c|}{ Acinetobacterspp. } & \multicolumn{2}{|c|}{ Other* } & \multicolumn{2}{|c|}{ Total } \\
\hline & CA 7 & HA 6 & CA 3 & HA 9 & CA 6 & HA 5 & CA 16 & HA 20 \\
\hline Ampicillin & 0 & 0 & 0 & 0 & 17 & 0 & 7 & 0 \\
\hline Amoxicillin-clavulanate & 17 & 20 & 67 & 33 & 67 & 0 & 47 & 22 \\
\hline Cefuroxime & 20 & 0 & 67 & 22 & 67 & 25 & 50 & 17 \\
\hline Ceftazidime & 100 & 100 & 67 & 78 & 83 & 50 & 88 & 79 \\
\hline Meropenem & 100 & 100 & 100 & 100 & 50 & 50 & 85 & 88 \\
\hline Gentamicin & 86 & 83 & 33 & 56 & 50 & 50 & 63 & 63 \\
\hline Tobramicin & 86 & 100 & - & - & - & - & - & - \\
\hline Doxycycline & 0 & 0 & 67 & 56 & 100 & 50 & 54 & 47 \\
\hline Co-trimoxazole & 0 & 0 & 0 & 0 & 33 & 50 & 14 & 13 \\
\hline Chloramphenicol & 20 & 0 & 33 & 22 & 67 & 0 & 43 & 13 \\
\hline Ciprofloxacin & 100 & 100 & 100 & 78 & 100 & 100 & 100 & 89 \\
\hline
\end{tabular}

CA, community-acquired infection; HA, hospital-acquired infection; "-", not done. *Other isolates include other Pseudomonas spp., (5),

Sphingomonas paucimobilis (2), Chryseobacterium spp. (2), Moraxella spp. (2). 
Table 8: Antimicrobial susceptibility (percentage) of Gram-positive bacteria causing bloodstream infection

\begin{tabular}{|c|c|c|c|c|c|c|}
\hline \multirow[t]{2}{*}{ Drug } & \multicolumn{2}{|c|}{ S. aureus } & \multicolumn{2}{|c|}{ E. faecium* } & \multicolumn{2}{|c|}{ E. faecalis* } \\
\hline & $\mathrm{CA} 13$ & HA 17 & $\mathrm{CA} 12$ & HA 9 & CA 9 & HA 6 \\
\hline Penicillin & 0 & 0 & 10 & 0 & 17 & 75 \\
\hline Ampicillin* & - & - & 25 & 11 & 100 & 100 \\
\hline $\begin{array}{l}\text { Amoxicillin- } \\
\text { clavulanate }\end{array}$ & 77 & 50 & 70 & $11+$ & 100 & 100 \\
\hline Cloxacillin & 92 & 88 & - & - & - & - \\
\hline Cefuroxime & 92 & 88 & - & - & - & - \\
\hline Ceftazidime & 85 & 80 & - & - & - & - \\
\hline Meropenem & 100 & 100 & - & - & - & - \\
\hline Gentamicin* & 100 & 81 & 67 & 33 & 56 & 67 \\
\hline Vancomycin* & 100 & 100 & 100 & 100 & 100 & 100 \\
\hline Erythromycin & 100 & 71 & 0 & 0 & 22 & 33 \\
\hline Doxycycline & 62 & 35 & 9 & 0 & 25 & 0 \\
\hline Co-trimoxazole & 69 & 63 & 27 & 0 & 67 & 40 \\
\hline Chloramphenicol & 85 & 73 & 50 & 11 & 38 & 33 \\
\hline Ciprofloxacin* & 77 & 81 & 42 & 22 & 100 & 100 \\
\hline Linezolid* & - & - & 100 & 100 & 100 & 100 \\
\hline $\begin{array}{l}\text { Quinupristin- } \\
\text { dalfopristin* }\end{array}$ & - & - & 100 & 100 & 0 & 17 \\
\hline
\end{tabular}

CA, community-acquired infection; HA, hospital-acquired infection; "-", not done. *E-test was used for susceptibility testing of enterococci against ampicillin, ciprofloxacillin, linezolid, quinupristin-dalfopristin, vancomycin and high-level gentamicin resistance. $\dagger$ Statistically significant difference in susceptibility among hospital-acquired and community-acquired isolates, $p<0.05$ by two-sided Fisher's exact test.

which was negatively associated with HIV infection $(7.1 \%$ versus $33.6 \%, P=0.005)$. HIV-positive children as a group did not receive more antimicrobial therapy (97.2\% versus 93.4\%, $P=0.082$ ). However, the given antimicrobial therapy for those with bloodstream infection was inappropriate more often than in HIV-negative children $(64.0 \%$ versus $40.2 \%, P=0.044)$. HIV-infection was a risk factor for death (Table 5, Figure 5), and associated with signifi-

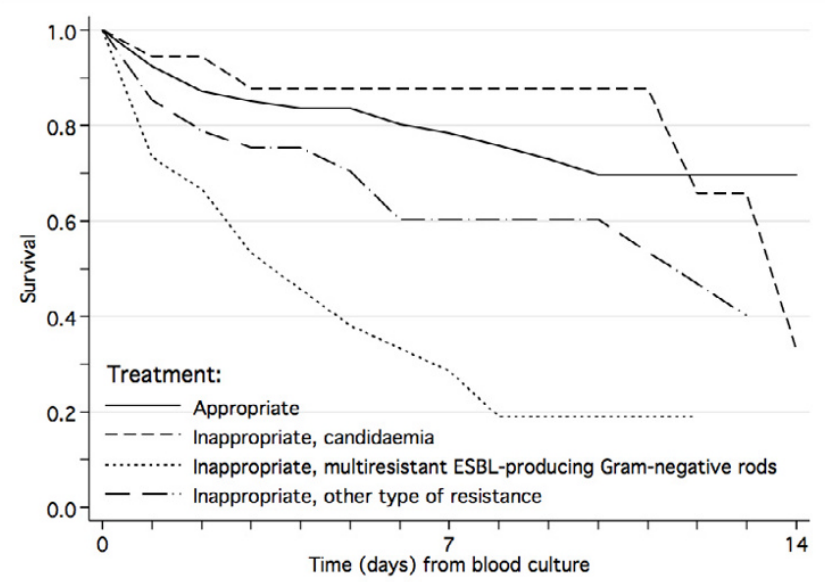

Figure 3

Impact of antimicrobial resistance on survival from laboratory-confirmed bloodstream infection. cantly longer hospital stay in those who survived (median 9 versus 6 days, $P<0.001)$.

\section{Malnutrition and other underlying conditions}

One sixth $(15.5 \%, 248 / 1603)$ of the patients were malnourished. Malnutrition was a risk factor for death (Table 5, Figure 6) and, in those who survived, it was associated with prolonged hospital stay (median 10 versus 6 days, $P$ $<0.001)$. Other underlying diseases were grouped into

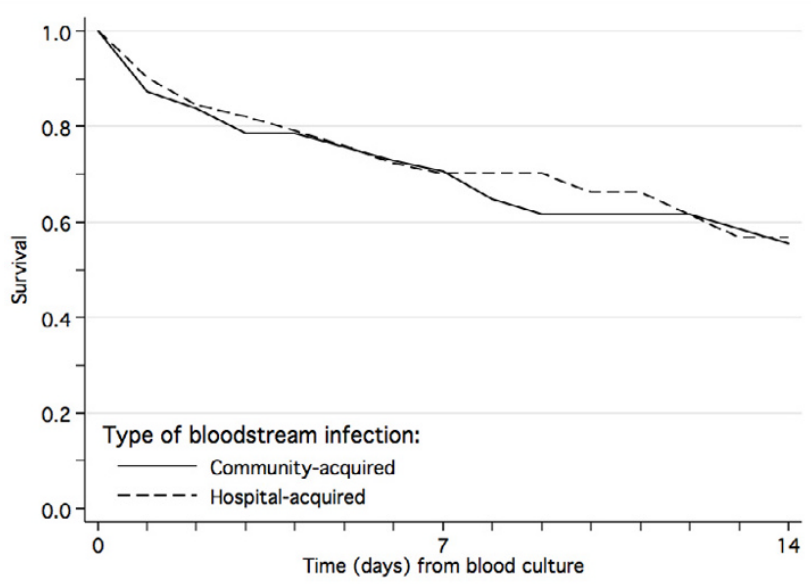

Figure 4

Survival from community-acquired and nosocomial laboratory-confirmed bloodstream infection. 


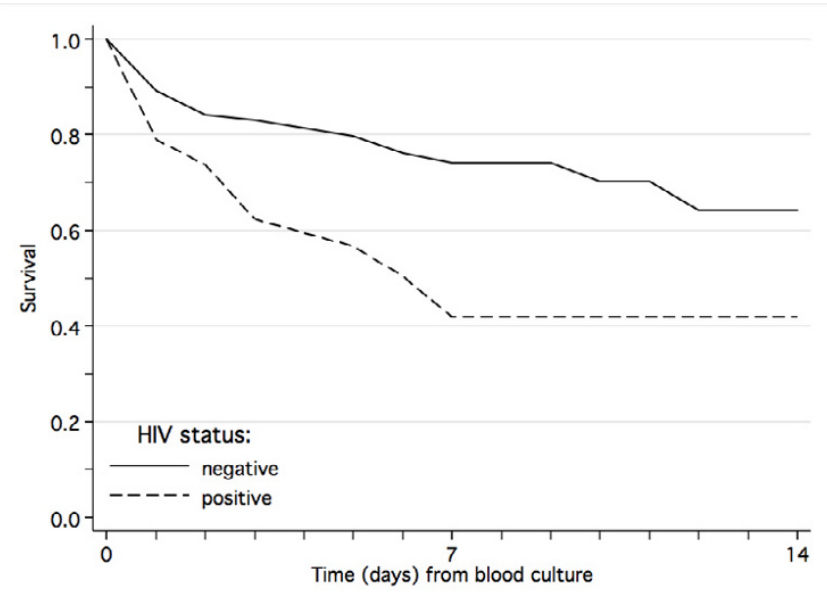

Figure 5

Impact of HIV co-infection on survival from laboratory-confirmed bloodstream infection.

infectious diseases, including tuberculosis ( $\mathrm{n}=97)$, hepatitis $(n=9)$ and congenital syphilis $(n=2)$ and non-infectious conditions, including sickle cell disease $(\mathrm{n}=36)$, cancer $(n=5)$, congenital heart disease $(n=38)$, asthma $(\mathrm{n}=5)$, Down's syndrome $(\mathrm{n}=11)$, cerebral palsy $(\mathrm{n}=$ $25)$, congenital malformations $(n=21)$, epilepsy $(n=10)$ and hemophilia $(\mathrm{n}=2)$. Other underlying infectious conditions than HIV were a significant risk factor for fatal outcome among all patients with clinically suspected systemic infection.

\section{Discussion}

Considering the frequent use of antimicrobials prior to blood-culture, the study probably underestimates the

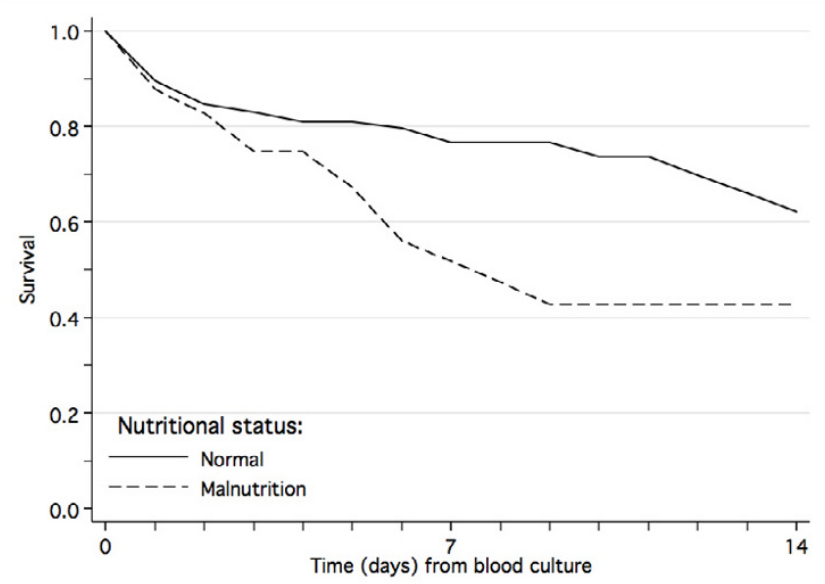

\section{Figure 6}

Impact of nutritional status on survival from laboratory-confirmed bloodstream infection. incidence of bloodstream infection, particularly episodes caused by fastidious organisms such as pneumococci and other organisms susceptible to commonly used antimicrobials. Nevertheless, the observed incidence of laboratory-confirmed bloodstream infection was high (13.9\%) and comparable to that of malaria (21.6\%). The therapeutic dilemma is evident, since bloodstream infection and malaria are difficult to distinguish based on clinical presentation $[4,5]$. While mortality was similar in bloodstream infection and malaria in a study from Rwanda in the 1980s [19], bloodstream infection carried much higher mortality than malaria in our study. As reported recently from Kenya [3], deaths from bloodstream infection outnumbered malaria deaths. The three times higher mortality among malaria-parasitemic patients with concomitant Gram-negative bloodstream infection underlines the relative importance of bloodstream infections. Prompt antimicrobial treatment is imperative for the survival of patients with bacterial bloodstream infection [20]. However, the impact of antimicrobial resistance on the clinical outcome has not been firmly ascertained, particularly in developing country settings $[6-8,21,22]$. The present study confirms that inappropriate treatment of bloodstream infections due to antimicrobial resistance predicts fatal outcome independently of underlying diseases, and is associated with longer duration of hospital stay in those who survive. While morbidity and cost are more sensitive measures of the impact of antimicrobial resistance in rich countries [7], our study demonstrates that the toll of antimicrobial resistance in Sub-Saharan Africa is quantifiable in loss of human lives.

The high rates of resistance in Gram-negative bacteria confirm previous findings [23]. However, the frequent use of antimicrobials prior to blood culture may have biased the findings by lowering the detection rate for organisms susceptible to commonly used drugs. It is grave that half of the klebsiella isolates, which are inherently resistant to ampicillin, are also resistant to gentamicin, since these two drugs are the most frequently used drugs for the treatment of bloodstream infections. The high incidence and case-fatality rate of klebsiella bloodstream infection in our study supports the findings by Zaidi and colleagues that klebsiella infections may be responsible for more than 0.3 million yearly neonatal deaths globally [22]. As reported elsewhere $[19,24,25]$, salmonella was the most frequent cause of bloodstream infections in older children. Nontyphoid salmonella cause a wide range of manifestations from self-limiting gastroenteritis to systemic infections such as bloodstream infections and sometimes meningitis associated with high mortality [26,27]. Bloodstream infection caused by Salmonella Typhimurium was associated with fatal outcome with comparable case-fatality rates as other major Enterobacteriaceae species, while bloodstream infection caused by $S$. Enteritidis was associ- 
ated with lower case-fatality rates in the same range as Gram-positive bloodstream infections. The relatively low case-fatality rate from bloodstream infection caused by $S$. aureus reflects the frequent use of cloxacillin and the near absence of methicillin-resistance. Bloodstream infection caused by E. faecium resistant to ampicillin and gentamicin is a potentially serious problem considering the widespread reliance on these drugs for treatment. The relatively low case-fatality rate from bloodstream infection caused by these organisms may reflect an inherently less virulent nature. However, it is also possible that some of the E. faecium isolates may have been skin contaminants, in which case the study may overestimate the incidence of bloodstream infection caused by this organism.

Hospital-acquired infection was associated with increased rate of resistance to cephalosporins in E. coli and co-trimoxazole in klebsiella, but it was not a risk factor for fatal outcome. Possibly, the definition used for hospitalacquired infection may have been too strict in the current study setting. The classification as hospital-acquired depended partly on the difference in time from admission to time of receiving the blood culture in the laboratory. Thus, any delay in obtaining or transporting blood-cultures may have led to some cases of community-acquired infection being wrongly classified as hospital-acquired. On the other side, it is less likely that cases of communityacquired infection have been misclassified as such, although we cannot rule out the possibility of underreporting of previous hospitalization.

Candida bloodstream infection was more frequent than reported from comparable low-income countries [22] and more in line with the trend in developed countries [28]. Candida bloodstream infection was not associated with HIV-infection or malnutrition. Candida was as common as a cause of community-acquired bloodstream infections $(10.2 \%)$ as hospital-acquired ones $(9.4 \%, P=0.837)$. The rare use of systemic antifungals may partly explain the high case-fatality rate associated with candida bloodstream infection.

The HIV prevalence $(16.8 \%)$ was higher than the country average (7\%) [29] and the estimate for Dar es Salaam $(10.8 \%)$ [30], but lower than in a study of children admitted to the same hospital in 1995-96 (19.2\%) [31], in which HIV status was verified by p24 antigen detection. The selection of study population (hospitalized children with fever) and the refusal by some parents to test their child may have influenced the HIV prevalence estimate.

Co-trimoxazole prophylaxis against Pneumocystis jirovecii pneumonia has been linked to resistance to this drug in HIV infected individuals [32-34]. In our study, HIV-positive subjects did receive inappropriate antibacterial ther- apy more frequently than HIV-negative, however, there was no significantly difference in resistance traits when analyzing for specific organism groups. Malnutrition predisposes patients for bacterial infection and is an underlying factor in more than half of under-five deaths [2]. Our study support the findings of previous studies that both malnutrition and HIV infection affect adversely the outcome of bloodstream infection $[2,34,35]$. The association between salmonella infection in HIV-infection is wellknown and has been attributed to a deficiency in the immune system that permits intracellular survival of the bacteria despite presumed adequate treatment $[24,25,36]$. While other infections such as Pneumocystis jirovecii pneumonia are important causes of death in HIV-infected children [37], our study suggest that bloodstream infection may contribute to the excess mortality in HIV-infected children in Sub-Saharan Africa [38].

In contrast to studies on adults from Tanzania and elsewhere [39], we isolated Mycobacterium tuberculosis from blood-culture from only one of 97 children with clinical tuberculosis. This finding indicates that blood-culture is a poor diagnostic tool for pediatric tuberculosis and underlines the experience from other studies that it is difficult to establish microbiological evidence to support the diagnosis of tuberculosis in children [40].

One limitation of the study is that clinical and outcome data were missing for some patients. Furthermore, we performed neither anaerobic culture nor repeat cultures to assess the significance of coagulase-negative staphylococci. The frequent use of antimicrobials before blood-culture and the small blood volume cultured, particularly from neonates $(1 \mathrm{ml})$, likely precluded the detection of pathogens, particularly fastidious organisms. The bloodculture system supported growth of pneumococci during quality testing in our laboratory. While available documentation by the start of our study supported use of the blood-culture system $[10,41]$, a later study indicated that it was not optimal for detection of pneumococci and $S$. aureus [42]. Sub-culturing on human blood agar plates may have interfered with the detection of pneumococci in the study. Human blood agar, prepared from expired banked blood, is widely used for bacterial isolation in developing countries, but has recently been demonstrated to be inferior to animal blood agars (sheep or horse blood) in detecting growth of common bacteria such as pneumococci, S. pyogenes and S. aureus. The use of human blood agar leads to reduced bacterial colony size, altered colony morphology and poor hemolysis [43]. The reasons for this are not fully understood, but may include remnants of antimicrobials consumed by the blood donor, antibodies or other unknown factors. While citrate in banked human blood has been considered a possible explanation for the inferiority of human blood agar for 
bacterial culture, Russell and colleagues found citrated sheep blood agar to be a practical and superior alternative to human blood agar [43]. While sheep and horse blood is often not readily available in many developing countries, goat or pig blood is more accessible and affordable and could be used for agar production [44]. A further reason for abandoning the use of human blood agar is the associated biohazard to the laboratory personnel due to frequent infection with HIV, hepatitis B and C among blood donors.

\section{Conclusion}

Our study shows that antimicrobial resistance predicts fatal outcome of bloodstream infection. While malaria can be treated with highly effective, inexpensive and widely available quinine, treatment of bacterial and fungal bloodstream infection is compromised by antimicrobial resistance and unavailability of expensive second-line antibacterial and systemic antifungal drugs. The finding that antimicrobial resistance, HIV-infection and malnutrition independently predicts fatal outcome not only call for renewed efforts to improve HIV care, nutrition and general living conditions for children, but also argue for increased focus on interventions to limit the selection pressure for resistant organisms such as education on rational use of antimicrobials, development of judicious guidelines for treatment strategies and adequate government regulation of drug quality and access to antimicrobial drugs [45].

\section{Competing interests}

The author(s) declare that they have no competing interests.

\section{Authors' contributions}

BB, KPM, WKU, BST, DSM, RJ, VM, MGT, SH, SYM and NL conceived and designed the study. The study clinicians were KPM, BST and other medical doctors in the Department of Pediatrics and Child Health. VM, MGT, BB and RJ analyzed the cultures. BB and MGT did the HIV rapid tests and MHP performed the HIV PCR. BB did the statistical analyses with contributions from $\mathrm{NL}, \mathrm{SH}$ and other authors. All authors contributed to the interpretation of the results and writing of the manuscript.

\section{Acknowledgements}

The study was supported by grant 100675 from the Norwegian Research Council and by funding from the Norwegian Council for Higher Education's Programme for Development Research and Education under project number 44003 PRO 42.2.91. We would like to thank all doctors, nurses, laboratory technicians and other staff at Muhimbili National Hospital who contributed to the study. We are particularly grateful to laboratory technologists Elizabeth Victor and Fredrick Kalokola-Kiiza for their support with blood-culturing and malaria microscopy, respectively, and doctor Gyri Vorren for assistance in data collection.

\section{References}

I. UNICEF: The state of the world's children 2006: Excluded and invisible. New York, The United Nations Children's Fund (UNICEF); 2005.

2. Bryce J, Boschi-Pinto C, Shibuya K, Black RE: WHO estimates of the causes of death in children. Lancet 2005, 365(9465): I | 47- I I52.

3. Berkley JA, Lowe BS, Mwangi I, Williams T, Bauni E, Mwarumba S, Ngetsa C, Slack MP, Njenga S, Hart CA, Maitland K, English M, Marsh $\mathrm{K}$, Scott JA: Bacteremia among children admitted to a rural hospital in Kenya. N Engl J Med 2005, 352(I):39-47.

4. Evans JA, Adusei A, Timmann C, May J, Mack D, Agbenyega T, Horstmann RD, Frimpong E: High mortality of infant bacteraemia clinically indistinguishable from severe malaria. Qjm 2004, 97(9):591-597.

5. Factor SH, Schillinger JA, Kalter HD, Saha S, Begum H, Hossain A, Hossain M, Dewitt V, Hanif M, Khan N, Perkins B, Black RE, Schwartz $B$ : Diagnosis and management of febrile children using the WHO/UNICEF guidelines for IMCI in Dhaka, Bangladesh. Bull World Health Organ 200I, 79(I 2): I096-I I05.

6. Okeke IN, Laxminarayan R, Bhutta ZA, Duse AG, Jenkins P, O'Brien TF, Pablos-Mendez A, Klugman KP: Antimicrobial resistance in developing countries. Part I: recent trends and current status. Lancet Infect Dis 2005, 5(8):48I-493.

7. Cosgrove SE: The relationship between antimicrobial resistance and patient outcomes: mortality, length of hospital stay, and health care costs. Clin Infect Dis 2006, 42 Suppl 2:S82-9.

8. Ramphal R, Ambrose PG: Extended-spectrum beta-lactamases and clinical outcomes: current data. Clin Infect Dis 2006, 42 Suppl 4:S164-72.

9. IMCI Integrated Management of Childhood IIIness. Model Chapter for Textbooks. 200I [http://www.who.int/child-adoles cent-health/integr.htm]. Geneva, World Health Organization

10. Archibald LK, McDonald LC, Addison RM, McKnight C, Byrne T, Dobbie H, Nwanyanwu O, Kazembe P, Reller LB, Jarvis WR: Comparison of BACTEC MYCO/F LYTIC and WAMPOLE ISOLATOR 10 (lysis-centrifugation) systems for detection of bacteremia, mycobacteremia, and fungemia in a developing country. J Clin Microbiol 2000, 38(8):2994-2997.

II. Collee JG, Marmion BP, Irvine R, Fraser AG, Simmons A: Mackie \& McCartney Practical Medical Microbiology. I4th edition. New York, Churchill Livingstone; 1996.

12. NCCLS: Performance Standards for Antimicrobial Disk Susceptibility Tests, Approved Standard M2-A7. 7th edition. Wayne, PA, USA, National Committee for Clinical Laboratory Standards; 1997.

13. Blomberg B, Jureen R, Manji KP, Tamim BS, Mwakagile DS, Urassa WK, Fataki M, Msangi V, Tellevik MG, Maselle SY, Langeland N: High rate of fatal cases of pediatric septicemia caused by gramnegative bacteria with extended-spectrum beta-lactamases in Dar es Salaam, Tanzania. J Clin Microbiol 2005, 43(2):745-749.

14. Brakstad OG, Maeland JA, Tveten Y: Multiplex polymerase chain reaction for detection of genes for Staphylococcus aureus thermonuclease and methicillin resistance and correlation with oxacillin resistance [published erratum appears in APMIS I 994 Mar; I02(3):24I]. Apmis 1993, I0I(9):68I-688.

15. Albert J, Fenyo EM: Simple, sensitive, and specific detection of human immunodeficiency virus type $I$ in clinical specimens by polymerase chain reaction with nested primers. J Clin Microbiol 1990, 28(7): I560-1564.

16. Grankvist O, Walther L, Bredberg-Raden U, Lyamuya E, Mhalu F, Gustafsson A, Biberfeld G, Wadell G: Nested PCR assays with novel primers yield greater sensitivity to Tanzanian HIV-I samples than a commercial PCR detection kit. J Virol Methods 1996, 62(2): |3|-|4|.

17. Stelling J, O'Brien TF: Whonet 5 Software. [http://www.who.int/ drugresistance/whonetsoftware/en/].

18. Stelling JM, O'Brien TF: Surveillance of antimicrobial resistance: the WHONET program. Clin Infect Dis 1997, 24 Suppl I:SI 57-68.

19. Lepage P, Bogaerts J, Van Goethem C, Ntahorutaba M, Nsengumuremyi F, Hitimana DG, Vandepitte J, Butzler JP, Levy J: Communityacquired bacteraemia in African children. Lancet 1987, I(8548): | 458-|46I.

20. Kumar A, Roberts D, Wood KE, Light B, Parrillo JE, Sharma S, Suppes R, Feinstein D, Zanotti S, Taiberg L, Gurka D, Kumar A, Cheang M: 
Duration of hypotension before initiation of effective antimicrobial therapy is the critical determinant of survival in human septic shock. Crit Care Med 2006, 34(6): 1589-1596.

21. Kim YK, Pai H, Lee HJ, Park SE, Choi EH, Kim J, Kim JH, Kim EC: Bloodstream infections by extended-spectrum beta-lactamase-producing Escherichia coli and Klebsiella pneumoniae in children: epidemiology and clinical outcome. Antimicrob Agents Chemother 2002, 46(5): | 481 - I 491.

22. Zaidi AK, Huskins WC, Thaver D, Bhutta ZA, Abbas Z, Goldmann DA: Hospital-acquired neonatal infections in developing countries. Lancet 2005, 365(9465): I I75-I I88.

23. Blomberg B, Mwakagile DS, Urassa WK, Maselle SY, Mashurano M Digranes A, Harthug S, Langeland N: Surveillance of antimicrobial resistance at a tertiary hospital in Tanzania. BMC Public Health 2004, 4(I):45.

24. Graham SM, Molyneux EM, Walsh AL, Cheesbrough JS, Molyneux ME, Hart CA: Nontyphoidal Salmonella infections of children in tropical Africa. Pediatr Infect Dis / 2000, I 9( I 2): I I89- I I96.

25. Bachou H, Tylleskar T, Kaddu-Mulindwa DH, Tumwine JK: Bacteraemia among severely malnourished children infected and uninfected with the human immunodeficiency virus- $I$ in Kampala, Uganda. BMC Infect Dis 2006, 6:160.

26. Saphra I, Winter JW: Clinical manifestations of salmonellosis in man; an evaluation of $\mathbf{7 7 7 9}$ human infections identified at the New York Salmonella Center. N Engl J Med 1957 256(24): I I 28- I I34.

27. Vaagland H, Blomberg B, Kruger C, Naman N, Jureen R, Langeland N: Nosocomial outbreak of neonatal Salmonella enterica serotype Enteritidis meningitis in a rural hospital in northern Tanzania. BMC Infect Dis 2004, 4(I):35.

28. Martin GS, Mannino DM, Eaton S, Moss M: The epidemiology of sepsis in the United States from 1979 through 2000. N Engl J Med 2003, 348(16): 1546-1554.

29. UNAIDS: AIDS epidemic update December 2005. Geneva Joint United Nations Programme on HIVIAIDS (UNAIDS), World Health Organization (WHO); 2005.

30. HIVIAIDS/STI Surveillance Report, January - December 2004. Dar es Salaam, National AIDS Control Programme; 2005.

31. Kawo G, Karlsson K, Lyamuya E, Kalokola F, Fataki M, Kazimoto T, Kitundu J, Msaky H, Munubhi E, Ostborn A, Bredberg-Raden U, Swai A, Mbise R, Msengi A, Mhalu F, Biberfeld G: Prevalence of HIV type I infection, associated clinical features and mortality among hospitalized children in Dar es Salaam, Tanzania. Scand J Infect Dis 2000, 32(4):357-363.

32. Wininger DA, Fass RJ: Impact of trimethoprim-sulfamethoxazole prophylaxis on etiology and susceptibilities of pathogens causing human immunodeficiency virus-associated bacteremia. Antimicrob Agents Chemother 2002, 46(2):594-597.

33. Jones N, Huebner R, Khoosal M, Crewe-Brown H, Klugman K: The impact of HIV on Streptococcus pneumoniae bacteraemia in a South African population. Aids 1998, I 2(16):2177-2 I84.

34. Madhi SA, Petersen K, Madhi A, Khoosal M, Klugman KP: Increased disease burden and antibiotic resistance of bacteria causing severe community-acquired lower respiratory tract infections in human immunodeficiency virus type I-infected children. Clin Infect Dis 2000, 3I(I): I70-I76.

35. Norton EB, Archibald LK, Nwanyanwu OC, Kazembe PN, Dobbie H, Reller LB, Jarvis WR, Jason J: Clinical predictors of bloodstream infections and mortality in hospitalized Malawian children. Pediatr Infect Dis J 2004, 23(2): I45-5I; discussion I5I-5.

36. Arthur G, Nduba VN, Kariuki SM, Kimari J, Bhatt SM, Gilks CF Trends in bloodstream infections among human immunodeficiency virus-infected adults admitted to a hospital in Nairobi, Kenya, during the last decade. Clin Infect Dis 200I, 33(2):248-256.

37. Lucas SB, Peacock CS, Hounnou A, Brattegaard K, Koffi K, Honde M, Andoh J, Bell J, De Cock KM: Disease in children infected with HIV in Abidjan, Cote d'Ivoire. BMJ 1996, 3 I 2(7027):335-338

38. Newell ML, Coovadia H, Cortina-Borja M, Rollins N, Gaillard P, Dabis F: Mortality of infected and uninfected infants born to HIV infected mothers in Africa: a pooled analysis. Lancet 2004, 364(944I): I236-I243.

39. McDonald LC, Archibald LK, Rheanpumikankit S, Tansuphaswadikul S, Eampokalap B, Nwanyanawu O, Kazembe P, Dobbie H, Reller LB, Jarvis WR: Unrecognised Mycobacterium tuberculosis bacter- aemia among hospital inpatients in less developed countries. Lancet 1999, 354(9 185): I I59-1 I63.

40. Kiwanuka J, Graham SM, Coulter JB, Gondwe JS, Chilewani N, Carty $\mathrm{H}$, Hart CA: Diagnosis of pulmonary tuberculosis in children in an HIV-endemic area, Malawi. Ann Trop Paediatr 200I, 2I(I):5-14.

4I. Archibald LK, den Dulk MO, Pallangyo KJ, Reller LB: Fatal Mycobacterium tuberculosis bloodstream infections in febrile hospitalized adults in Dar es Salaam, Tanzania. Clin Infect Dis 1998, 26(2):290-296.

42. Vetter E, Torgerson C, Feuker A, Hughes J, Harmsen S, Schleck C, Horstmeier C, Roberts G, Cockerill F 3rd: Comparison of the BACTEC MYCO/F Lytic bottle to the isolator tube, BACTEC Plus Aerobic F/bottle, and BACTEC Anaerobic Lytic/ 10 bottle and comparison of the BACTEC Plus Aerobic F/bottle to the Isolator tube for recovery of bacteria, mycobacteria, and fungi from blood. J Clin Microbiol 200I, 39( I 2):4380-4386.

43. Russell FM, Biribo SS, Selvaraj G, Oppedisano F, Warren S, Seduadua A, Mulholland EK, Carapetis JR: As a bacterial culture medium, citrated sheep blood agar is a practical alternative to citrated human blood agar in laboratories of developing countries. J Clin Microbiol 2006, 44(9):3346-335।.

44. Anand C, Gordon R, Shaw H, Fonseca K, Olsen M: Pig and goat blood as substitutes for sheep blood in blood-supplemented agar media. J Clin Microbiol 2000, 38(2):59I-594.

45. Okeke IN, Klugman KP, Bhutta ZA, Duse AG, Jenkins P, O'Brien TF, Pablos-Mendez A, Laxminarayan R: Antimicrobial resistance in developing countries. Part II: strategies for containment. Lancet Infect Dis 2005, 5(9):568-580.

\section{Pre-publication history}

The pre-publication history for this paper can be accessed here:

\section{http://www.biomedcentral.com/1471-2334/7/43/prepub}

Publish with Bio Med Central and every scientist can read your work free of charge

"BioMed Central will be the most significant development for disseminating the results of biomedical research in our lifetime."

Sir Paul Nurse, Cancer Research UK

Your research papers will be:

- available free of charge to the entire biomedical community

- peer reviewed and published immediately upon acceptance

- cited in PubMed and archived on PubMed Central

- yours - you keep the copyright
BioMedcentral 